\title{
Persistence behavior in African trypanosomes during adipose tissue colonization
}

\section{Sandra Trindade}

Instituto de Medicina Molecular, Faculdade de Medicina, Universidade de Lisboa

\section{Mariana De Niz}

Instituto de Medicina Molecular, Faculdade de Medicina, Universidade de Lisboa

\section{Mariana Sequeira}

Instituto de Medicina Molecular, Faculdade de Medicina, Universidade de Lisboa https://orcid.org/0000-0003-1486-5403

\section{Tiago Bizarra-Rebelo}

Instituto de Medicina Molecular, Faculdade de Medicina, Universidade de Lisboa

\section{Fabio Bento}

Instituto de Medicina Molecular, Faculdade de Medicina, Universidade de Lisboa

\section{Mario Dejung}

Institute of Molecular Biology

\section{Joao Ferreira}

Instituto de Medicina Molecular, Faculdade de Medicina, Universidade de Lisboa

\section{Falk Butter}

Institute of Molecular Biology

\section{Frederic Bringaud}

University of Bordeaux

\section{Erida Gjini}

Instituto Gulbenkian de Ciência

\section{Luisa Figueiredo ( $\square$ Imf@medicina.ulisboa.pt)}

Instituto de Medicina Molecular, Faculdade de Medicina, Universidade de Lisboa https://orcid.org/0000-0002-5752-6586

\section{Article}

Keywords: Trypanosoma brucei, persistence, sleeping sickness

Posted Date: May 20th, 2021

DOI: https://doi.org/10.21203/rs.3.rs-499897/v1 
License: (c) (i) This work is licensed under a Creative Commons Attribution 4.0 International License. Read Full License 


\section{Persistence behavior in African trypanosomes during 2 adipose tissue colonization}

3

4 Sandra Trindade ${ }^{1}$, Mariana De Niz ${ }^{1}$, Mariana Costa-Sequeira ${ }^{1}$, Tiago Bizarra-Rebelo ${ }^{1}$, Fábio 5 Bento $^{1 \not}$, Mario Dejung ${ }^{2}$, João Ferreira ${ }^{1}$, Falk Butter ${ }^{2}$, Frédéric Bringaud ${ }^{3,4}$, Erida Gjini ${ }^{5 *^{*}}$ \& Luisa M. 6 Figueiredo ${ }^{1 *}$.

7

8

$11{ }^{3}$ Laboratoire de Microbiologie Fondamentale et Pathogénicité (MFP), Université de Bordeaux,

12 CNRS UMR-5234, Bordeaux, France

$13{ }^{4}$ Centre de Résonance Magnétique des Systèmes Biologiques (RMSB), Université de Bordeaux,

14 CNRS UMR-5536, Bordeaux, France

15 Instituto Gulbenkian de Ciência, 2780-156 Oeiras, Portugal

$16{ }^{\mathrm{x}}$ Current address: Institute of Molecular Biology, Mainz, Germany

$17 \quad{ }^{\ddagger}$ Current address: Center for Computational and Stochastic Mathematics, Instituto Superior 18 Técnico, Universidade de Lisboa, 1049-001 Lisbon, Portugal.

19 *Luisa M. Figueiredo, Erida Gjini.

20 Email: Imf@medicina.ulisboa.pt and erida.gjini@tecnico.ulisboa.pt
21

22

23

24

25

26

27

28

29

30

31

32 
Persistence is an important and ancient evolutionary adaptive mechanism used by several organisms to survive environmental changes. During its life cycle Trypanosoma brucei, the causative agent of sleeping sickness, inhabits several microenvironments, including the adipose tissue. Here we used a mathematical model to investigate how this large parasite reservoir contributes to the global parasite population dynamics. By modeling the total number of parasites and the proportion of transmissible forms in the blood and the adipose tissue during an infection, we estimated that adipose tissue parasites proliferate more slowly. Intravital microscopy of parasites stained with CellTrace ${ }^{\mathrm{TM}}$ Violet confirmed that adipose tissue forms divide twice slower than the blood counterparts. Consistent with a reduced growth, proteome analysis revealed that adipose tissue forms undergo a metabolic adaptation and downregulate proteins involved in translation. Quantification of protein synthesis using L-Homopropargylglycine confirmed that this rate is $24 \%$ lower in adipose tissue parasites. We propose that in adipose tissue, $T$. brucei acquire a persistence-like behavior, which could contribute to disease chronicity and treatment failure.

Persistence was first described in bacteria and has been well characterized in several different bacterial species ever since ${ }^{1}$. Persisters are a subgroup of viable cells of a population characterized by growth arrest or slower growth, altered metabolism, downregulation of protein synthesis and increased resistance to environmental threats such as immunological attack and drug treatment. This condition is reversible and persisters can return to a more proliferative state. Among parasitic protozoa, the existence of persisters has only been recently documented, and knowledge on the biological roles of these parasites subpopulations is still scarce ${ }^{2}$. Toxoplasma gondii, a protozoan parasite that infects most species of warm-blooded animals, upon host invasion, disseminate into tissues and differentiate into persistent metabolically active, slow grower bradyzoites as a strategy to overcome nutrient limitations and host immune pressure ${ }^{3}$. Persistence can also be triggered by temporary exposure to external stresses. $P$. falciparum, for instance, spontaneously develops into dormant ring stages with a distinct metabolic state upon artemisinin exposure ${ }^{4}$ Importantly, these persistent parasitic protozoa are refractory to artemisinin-based drugs promoting drug treatment failure ${ }^{2}$.

Trypanosoma brucei parasites, the causative agents of Human African Trypanosomiasis (HAT), have a complex life cycle that oscillates between a tsetse vector and a mammalian host. During the mammal infection, parasites invade and occupy the interstitial spaces of different organs, such as the central nervous system, adipose tissue and skin ${ }^{5}$. Transmission between hosts is ensured by the quiescent non-dividing stumpy forms and metacyclic forms. These non-replicative forms are pre-adapted to survive in the tsetse midgut and the mammalian host environments, respectively. Stumpy forms are also important to reduce global parasite load in the mammalian host. Similar to what has been described in bacteria, these parasites change their metabolism and reduce their protein synthesis ${ }^{6,7}$.

A different source of heterogeneity within a $T$. brucei population stems from the adaptation to the tissue environment. We have previously shown that, in a mouse infection, adipose tissue is

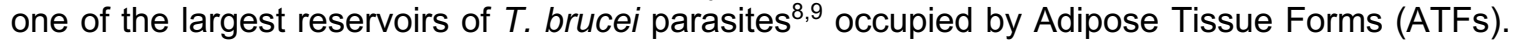
These forms are functionally different from the bloodstream counterparts (BSFs), with a metabolism apparently adapted to catabolize fatty acids and are capable of re-infecting the blood circulation, showing that they are not terminally differentiated to live exclusively in the adipose tissue ${ }^{8}$.

In this work, we use mathematical modelling to determine key parameters that govern $T$. brucei chronic infections taking into account the existence of tissue reservoirs. We experimentally confirmed the prediction of the model for differences between reservoirs, showing that the adipose tissue harbors slow growing parasites that synthesize proteins at a lower rate. Together with previous knowledge that ATFs are metabolically distinct from the blood counterparts ${ }^{8}$, we propose the existence of persister-like cells in the adipose tissue population. This finding has important implications in our understanding of drug resistance and relapses, and reveals that adipose tissue acts as a reservoir of quieter parasites that may cause less pathology and thus contribute to disease chronicity. 


\section{Results}

\section{Parasite dynamics differ between blood and adipose tissue}

Adipose tissue is a major parasite reservoir during the acute and the chronic stages of the disease in mice. We previously observed that, on day 6 of infection, the adipose tissue is mainly populated by slender forms, while the blood has a majority of transmissible stumpy forms ${ }^{8}$. Whether the parasite load remains high in the adipose tissue and the proportion of slender and stumpy forms varies in the two tissues, throughout infection, remains unknown. To answer this question, we infected mice with a pleomorphic GFP.:PAD1 utr reporter parasite line that allowed us to follow both the total parasite load, and the proportion of transmissible forms (because stumpy forms are GFPexpressers) in each tissue for over 28 days of infection ${ }^{8}$. Mice were sacrificed on 17 days of infection and the total number of parasites per organ (parasite load) was quantified by qPCR from genomic DNA isolated from blood and perfused gonadal adipose tissue. BSFs and ATFs were also isolated from both tissues to assess the percentage of stumpy forms by flow cytometry.

In general, both tissues remained highly parasitized throughout infection. When we compared parasite density (parasites/mg), the gonadal adipose tissue was on average $\sim 6$-fold more concentrated than blood. When we compared the parasite load (number of parasites in whole organ), the blood had on average $\sim 1.4$-fold more parasites than gonadal adipose tissue (Supplementary Table S1). As previously observed ${ }^{8,10,11}$, during the first week of infection, the blood showed a larger range of parasite load with a peak in total number of parasites on day $6\left(\sim 10^{8}\right.$ parasites) and a subsequent trough on day $8\left(\sim 2 \times 10^{3}\right.$ parasites $)$. During this period, the number of parasites in the gonadal adipose tissue varied less $\left(10^{4}-10^{7}\right.$ parasites) (Fig. $1 \mathrm{~A}$ and Supplementary Table S1). These results showed an initial slower colonization of the adipose tissue and a less pronounced reduction of this population after day 6 of infection, as revealed by the roughly $1,000-$ fold more parasites in the gonadal adipose tissue than in the blood at days 7 and 8 post infection. From day 13 forward, the total number of parasites was less variable and the pattern more similar between tissues $\left(\sim 10^{6}-10^{7}\right.$ parasites), with the blood having on average around 3 -fold more parasites than the gonadal adipose tissue (Fig. 1A and Supplementary Table S1). Since parasite load was not measured daily, we cannot discard the possible existence of other troughs, although this is highly unlikely because others have shown that parasitemia remains relatively high after day 12 of infection ${ }^{10}$.

Next, we assessed if the proportion of replicative (slender) and non-replicative transmissible (stumpy) forms is different between the two tissues throughout infection. Parasites were isolated from blood and gonadal adipose tissue and flow cytometry was used to calculate the proportion of parasites that expressed GFP (stumpy reporter gene driven by 3'UTR of PAD1) ${ }^{12}$. In general, we observed that in the adipose tissue, the average percentage of stumpy forms was lower (Wilcoxon Signed Rank test, $p<0.0001$ ) and more evenly distributed than in the blood. While the adipose tissue percentage of stumpy forms is normally distributed (Kolmogorov-Smirnov Test, $p>0.1$ ) with a mean of $52 \%$ and a standard deviation of $31 \%$, the blood percentage of stumpy forms follows a non-normal distribution (Kolmogorov-Smirnov Test, $p<0.0001$ ) with a mean of $79 \%$ and a standard deviation of $28 \%$ (Fig. 1B). Indeed, except for day 4 , the blood was always richer in stumpy forms than the gonadal adipose tissue. Interestingly, for the first two weeks of infection, the accumulation of stumpy forms in the adipose tissue proceeds more slowly than in the blood. This is consistent with the fact that in most days of the infection, the parasite load in blood is higher than in adipose tissue (Fig. 1A). When we compare data from an individual mouse, it is also possible to observe that the proportion of stumpy forms can be dramatically different between the two microenvironments (Fig. 1C), indicating that within a single animal there are significant differences in the proportion of stumpy forms across tissues.

We conclude that although blood and gonadal adipose tissue are highly parasitized throughout infection, the dynamics of the number of parasites and the proportion of slender/stumpy forms are different between the two compartments. Relative to the blood, the adipose tissue is poorer in stumpy forms. These results suggest an influence from the tissue microenvironment and the existence of an important barrier (likely, the vasculature) that prevents homogenization of the two parasite populations. 


\section{Infection modeling anticipates differences in parasite growth between tissues}

To quantify the infection processes in more detail, and investigate parameter differences between microenvironments, we built a mathematical model that describes parasite growth, differentiation, antigenic variation dynamics and the interplay with host immunity. The model was based on a previous mathematical formulation ${ }^{13}$, but was now extended to include two connected in-host compartments: blood and adipose tissue, with parasite migration between them (Fig. $2 \mathrm{~A}$ and Supplementary Model Information). The primary aim of the model was to integrate under the same mechanistic framework the empirical data on the parasite density and the proportion of stumpy forms across the two compartments over time. Parasite density was used instead of total number of parasites to avoid misinterpretations derived from the observed fluctuations in the adipose tissue weight (Supplementary Table S1). While some model parameters were assumed fixed at biologically reasonable values (Supplementary Table S2), other key infection parameters were estimated (Table 1).

To test for plausible parameter differences between the blood and adipose tissue, we considered three nested versions of the model. In model 1 (the null hypothesis), no infection parameter differences between compartments were assumed (except for the blood exclusive onset of infection and antigen switching); in model 2, the parasites may grow at different rates in each compartment; in model 3, in addition to growth rates, differentiation parameters could also vary across the two tissues. Although the model structure is flexible enough to explore many more hypotheses about parameter differences between compartments, we limited our analysis to these three basic formulations, differing sequentially in just one parameter, which are the simplest ones to start with, and easier to validate with additional experiments.

We fitted each model dynamically to the experimental data under a Bayesian framework, and then compared their results based on the Deviance Information Criterion (DIC) ${ }^{14}$, the likelihood ratio $^{15}$, as well as the visual inspection of the $95 \%$ credible envelopes for the infection trajectories. Model fits generated model-specific estimates for infection parameters and corresponding predictions for the inter-coupled infection dynamics between blood and adipose tissue, even though the models are realistically close to each other (Table 1 and Supplementary Model Information). The DIC criterion and the likelihood ratio test were the statistical indicators that allowed comparing how well the models fit the raw data, balancing quality of fit with model complexity. Typically, models with a smaller DIC are favored. In addition, a large likelihood ratio would favor the more complex model while a small likelihood ratio would favor the simpler model. For this specific dataset, model 2 was favored over model 1 by both the above criteria. The model was able to capture accurately the global parasite dynamics across the blood and adipose tissue over 28 days (Fig. 2B and Supplementary Model Information). Further adding an extra layer of complexity, by allowing variation of the differentiation parameter across the two compartments (model 3 ), produced largely overlapping estimates for these infection traits in blood and adipose tissue and did not significantly improve the model fit (Supplementary Table S3).

The key parameter change between the null model 1 and favored model 2 is the difference in parasite growth rates between the two compartments. While model 1 estimated a single growth rate for the entire population as a null-hypothesis, model 2 estimated a doubling time of 7 hours for the parasite population in the blood and 13.7 hours for parasites residing in the adipose tissue. This observation is in good agreement with previous modeling where bloodstream form parasites were estimated to divide every 6 hours ${ }^{16}$. The inferred $50 \%$ lower growth rate of slender forms in the adipose tissue (Table 1), enabled model 2 to better describe the observed slow buildup of stumpy forms over time in this tissue. This indicates that allowing for heterogeneous replication of slender form parasites between the blood and adipose tissue is both necessary and sufficient to capture the global patterns in the experimental infection data over 28 days (Fig. 2B and Supplementary Model Information).

Overall, going beyond single snapshot analysis, and accounting for the inter-dependent temporal structure and feedbacks between infection variables, we were able to integrate in a dynamic model the experimental data on $T$. brucei parasite density and the proportion of stumpy forms in blood and adipose tissue of infected mice. The best-fitting model indicates that the replication of parasites residing in the adipose tissue is 2 -fold slower than the ones in the blood.

\section{Proteome analysis suggests downregulated protein translation in ATFs}

To experimentally validate the model prediction that slender forms in the adipose tissue grow twice longer than in the blood, we started by comparing the proteome of slender forms isolated 
from blood and gonadal adipose tissue. Given that the proteome of stumpy and slender forms is different ${ }^{17}$, and stumpy forms are present in unequal amounts in the two tissues, we chose to infect mice with a monomorphic strain, which does not form stumpy forms. Five days post-infection, parasites were isolated from blood and adipose tissue and processed for proteome analysis.

We identified a total of 2693 protein groups among ATF and BSF parasites (Supplementary Dataset S1), from which $6 \%$ were differentially expressed, namely, 54 protein groups were upregulated and 112 protein groups were downregulated in the ATFs (Fig. 3A). The relative abundances of the differentially expressed protein groups showed high consistency between replicates (Fig. 3B), suggesting that the differences detected between ATF and BSF parasites are reproducible between infections in different animals.

To identify which processes may be downregulated in ATFs, we performed a Gene Ontology (GO) term enrichment analysis of the downregulated proteins (Fisher's exact test, $\mathrm{p} \leq 0.05)$. The biological process ontology showed significant depletion of two GO terms, namely, translation and ribosome biogenesis (Supplementary Table S4). Among those, the most significant alteration that took place in ATFs was the down-regulation of proteins involved in translation (Fig. $3 \mathrm{C}$ and Supplementary Dataset S1 and Table S4). 96 ribosomal proteins of 208 annotated protein groups within this GO term were down-regulated in the ATF proteome. Ribosome biogenesis, a pivotal process for protein translation was also diminished. A curated analysis of the proteins revealed that multiple ribosomal proteins of both $40 \mathrm{~S}$ and $60 \mathrm{~S}$ ribosomal subunits were downregulated. Interestingly, despite not annotated to any of the obtained GO terms, the second largest subunit of the RNA polymerase I was also downregulated (Tb927.11.630, fold change of 0.5 ) suggesting a lower transcription of ribosomal gene units (Supplementary Dataset S1 and Table S4). These data strongly suggest that ATFs undergo a generalized reduction in the machinery responsible for synthesizing proteins.

GO term analysis of the proteins upregulated in ATFs (Supplementary Table S4) suggests that these parasites are metabolically distinct from BSFs, which is consistent with our previous transcriptomic and biochemical characterization ${ }^{8}$. Interestingly, the most significant category of upregulated proteins comprised purine metabolism ( 5 of the 54 upregulated protein groups) (Fig. $3 \mathrm{C}$ ). The upregulation of the purine nucleoside transporter, with high affinity for adenosine, and adenosine transporter 2 ((Tb927.9.7470 and Tb927.2.6150, respectively, fold change 6.8), together with inosine-adenosine-guanosine-nucleosidehydrolase (Tb927.3.2960, fold change 1.8) and adenine phosphoribosyltransferase (Tb927.7.1780, fold change 2.0) (Fig. 3C and Supplementary, Dataset S1 and Table S4), could culminate in increased cellular levels of adenosine monophosphate (AMP) and consequently stimulate the ATP production and/or affect the AMP/ATP ratio.

Mitochondrial pathways involved in central metabolism are also upregulated in ATFs with evidence in the proteome of upregulated enzymatic reactions involved in ATP metabolism (13 of the 54 upregulated genes). Namely, the upregulation of two of the three enzymes composing the mitochondrial acetate production pathway, which is involved in ATP production in the mitochondrion ${ }^{18-20}$, i.e. the pyruvate dehydrogenase complex (PDH; Tb927.3.1790, Tb927.10.12700 and Tb927.10.2350, fold change of 2.0) and acetate:succinate CoA-transferase (ASCT; Tb927.11.2690, fold change of 2.7). Also, enzymes of the glutamine catabolic pathway, which has been reported to be functional in $\mathrm{BSFs}^{21}$ and participate in ATP production, are upregulated in ATFs. This includes glutamate dehydrogenase (Tb927.9.5900, fold change 2.8), which converts glutamate to $\alpha$-ketoglutarate and the $\alpha$-ketoglutarate dehydrogenase complex (Tb927.11.11680, Tb927.11.1450 and Tb927.11.9980, fold changes 1.9, 3.4 and 3.1, respectively) (Supplementary Dataset S1). It is noteworthy that the proline degradation pathway, which includes the upregulated delta-1-pyrroline-5-carboxylate dehydrogenase (Tb927.10.3210, fold change 1.9), also produces glutamate that can then be converted into $\alpha$-ketoglutarate as well. In addition, the proteome of ATFs suggested a concomitant expanded use by the mitochondrial $F_{1} / F_{0}$-ATP synthase of the ATP produced in the mitochondrion. Indeed, the absent expression of any component of the oxidative phosphorylation pathway together with the increased expression levels of four mitochondrial $\mathrm{F}_{1} / \mathrm{Fo}_{\mathrm{o}} \mathrm{ATP}$-synthase subunits pointed towards a reverse mode of action with a concomitant ATP hydrolyzation in these parasite forms. Namely, the $F_{1}$ complex subunit gamma (Tb927.10.180, fold change 1.7), the Fo complex subunit B (Tb927.5.1710, fold change of 1.8), the subunit 5 (Tb927.10.8030, fold change 1.6) and the putative ATP synthase subunit (Tb927.5.3090, 
fold change 2.3) (Supplementary Dataset S1) of the mitochondrial $F_{1} / F_{0}-A T P-s y n t h a s e$ showed increased expression levels.

Curiously, evidence for an increased oxidative stress response was also observed in the adipose tissue parasites (oxidation-reduction process group) (Fig. $3 \mathrm{C}$ ), with the upregulation of iron/ascorbate oxidoreductase family protein and iron superoxide dismutase A (Tb11.v5.0215 and Tb927.5.3350, fold changes 6.3 and 1.8, respectively) (Supplementary Dataset S1), probably as part of the adaptation process to the new environment.

In summary, the proteome analysis revealed that the slender forms that live in the adipose tissue and blood present significantly different proteomes. Gene function analysis revealed that ATFs have a reduced protein synthesis and a remodeled metabolism that is oriented towards AMP/ATP production and increased mitochondrial activity.

\section{ATFs have reduced protein synthesis}

Next, we investigated whether the protein synthesis of adipose tissue parasites is lower than in blood parasites. We measured protein synthesis by labeling newly synthesized proteins with a methionine analog, Click-i ${ }^{\circledR}$ Homopropargylglycine (HPG), in parasites isolated from each of the two tissues. Levels of labelled nascent proteins in BSFs and ATFs were measured by flow cytometry. Given that this method has never been optimized for T. brucei, first we tested the linearity of the assay by cultivating bloodstream forms in three increasing concentrations of HPG $(25 \mu \mathrm{g}, 50 \mu \mathrm{g}$ and $100 \mu \mathrm{g})$ for 30 minutes. The Mean Fluorescence Intensity (MFI) measured by flow cytometry showed a dose-dependent response in which the levels of labelled proteins increased

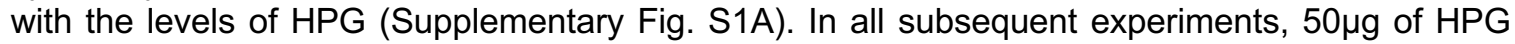
were used. Next, we compared the protein synthesis between BSFs and Procyclic Forms (PCFs). Previous studies using ${ }^{35} \mathrm{~S}$-methionine-labelling, showed that the protein synthesis of PCFs is 25$37 \%$ lower than $\mathrm{BSFs}^{22}$. Our HPG-labelling assay revealed that PCFs incorporated on average $74 \%$ less HPG than BSFs (Supplementary Fig. S1B), which is consistent although more pronounced than previously reported ${ }^{22}$. Differences in the parasite strains, medium composition and growth conditions may explain the observed discrepancies between the two studies. To confirm that HPG intensity was a result of its incorporation into proteins and not of free HPG inside the parasites, we inhibited protein synthesis of cultured parasites with cycloheximide. We observed that the levels of HPG intensity dropped to $7 \%$ of condition without cycloheximide, validating our assay (Supplementary Fig. S1C).

To compare the protein synthesis of parasites from different tissues ex vivo, we infected mice with the same monomorphic strain that is incapable of differentiating to stumpy forms, a life cycle stage that is well known to also have a reduced protein synthesis. Mice were sacrificed on day 5 post-infection and parasites were isolated from blood and gonadal adipose tissue in parallel. In each of the six independent experiments, parasites from 4-5 mice were pooled prior to HPG labelling. After incubation for 30 minutes with HPG, parasites were washed and analyzed as indicated in Fig. 3D. Flow cytometry revealed that the intensity of HPG signal was $24 \%$ lower in parasites isolated from adipose tissue than from blood (Fig. 3E). Although the absolute levels of HPG-labelled proteins showed some variability between independent experiments, the intensity of HPG-labelled proteins was always lower in ATFs than BSFs (with a range of reduction between $14 \%-35 \%$ ) (Fig. 3F), indicating that adipose tissue forms have a reduced protein synthesis (Wilcoxon Signed Rank Test, $p<0.05$ ).

\section{Parasites in the adipose tissue proliferate more slowly than in the blood}

In many eukaryotes, a reduced rate of protein synthesis is normally associated to slower growth ${ }^{23,24}$. Our modeling analysis indeed suggested that in the adipose tissue parasites replicate every $13 \mathrm{hr} 42 \mathrm{~min}$, unlike $7 \mathrm{hr} 6 \mathrm{~min}$ in the blood (Table 1). To test whether ATFs replicate more slowly than BSFs, we started by analyzing the cell cycle pattern of ATFs vs BSFs. First, we used intravital imagining to compare the number of kinetoplasts/nuclei $(\mathrm{KsNs})^{25}$ in parasites present in blood and adipose tissue. Intravenous administration of Hoechst to mice infected for 5 days with a monomorphic strain (Fig. 4A and Supplementary Movie S1) revealed that ATFs have on average a significantly higher percentage of parasites presenting 1K1N (cells in G1, early S phase or G0arrested cells) (73.6\% vs $60.1 \%$ in BSFs) and $2 \mathrm{~K} 1 \mathrm{~N}$ (G2 and mitotic cells) $(17.1 \%$ vs $11.6 \%$ in BSFs) configurations. The most significant difference was that ATFs have a lower percentage of $2 \mathrm{~K} 2 \mathrm{~N}$ parasites (post-mitotic) $(9.3 \%$ vs $28.3 \%$ in BSFs), suggesting that in a snapshot of the ATF population it is less likely to find parasites that just underwent cell division than in a BSF population, 
which would be expected if ATFs proliferate more slowly (Fig. 4B). An ex vivo parallel analysis on parasites previously isolated from blood and adipose tissue, showed similar results, indicating that ex vivo cell cycle analysis is a valid method for future studies (Supplementary Fig. S2A).

To further confirm the differences of cell cycle between parasites in the blood and adipose tissue, we measured the DNA synthesis ( $S$ phase) in parasites isolated from both tissues. For that, mice infected for five days with the same monomorphic strain were intravenously injected with 5ethynyl-2-deoxyuridine (EdU), a thymidine analogue that is actively incorporated into newly synthesized $\mathrm{DNA}^{26}$. After 30 minutes, blood and adipose tissue parasites were isolated from a pool of two mice and analyzed by microscopy ex vivo for the percentage of EdU positive cells (Fig. 4C). The ATF population showed on average a lower percentage of labeled cells than BSFs $(15.8 \%$ vs $24.6 \%$, respectively) (Fig. 4D), indicating that the ATF population has fewer cells entering DNA synthesis. In parallel, we performed an ex vivo EdU labeling of parasites previously isolated from a pool of two mice infected for the same period with the same strain. EdU labeling ex vivo corroborated our observations in vivo, indicating that the parasite isolation protocol does not affect DNA synthesis (Supplementary Fig. S2B). The profile of KsNs together with the EdU incorporation analysis, clearly indicate that the cell cycle of parasites in the fat and blood is different. ATFs appear to spend more time in $\mathrm{G} 1$ than their blood counterparts.

Finally, we determined the doubling time of parasites in blood and adipose tissue. For that, mice were infected with monomorphic parasites previously labelled in vitro with CellTrace ${ }^{\mathrm{TM}}$ Violet (CTV), a fluorescent dye that binds to all free amines inside cells. Every time a cell divides, the amount of CTV is halved ${ }^{27}$, which allows the estimation of doubling time by scoring the fluorescence intensity in labeled parasites (see Experimental Procedures). 2 days post-infection, we performed intravital microscopy of the blood and adipose tissue populations (Fig. 4E) and we measured the intensity of the CTV-positive cells. The adipose tissue revealed a more heterogeneous population with mean fluorescence intensities oscillating between 3.7 and 489.3 , whereas the bloodstream forms mean fluorescence intensity oscillated between 0.1 and 72.6 (Fig. 4F). Indeed, $40 \%$ of the studied adipose tissue population displayed exclusive high intensities of CTV. The calculated doubling time of BSFs was 6.2 hours, which is in good agreement with previous reports for both in vivo and in vitro growth of BSFs 28,29 . In contrast, the doubling time of ATFs was 12.4 hours, i.e. twice longer than BSFs (Fig. 4G). To exclude that the staining background detected in vivo was influencing our analysis, we also did the analysis ex vivo, in two isolated labeled populations. The ex vivo data clearly corroborated the intravital microscopy observations: BSFs presented a doubling time of 6.2 hours contrasting to ATFs 11.6 hours (Supplementary Fig. S2C).

Overall, our results show that in the adipose tissue, the slender form parasites have a reduced protein synthesis and a concomitantly reduced growth rate. Together with our initially reported observation that ATFs are metabolically distinct from bloodstream counterparts, we conclude that colonization of adipose tissue triggers a persistent behavior in T. brucei.

\section{Discussion}

The occurrence of non-dividing or slow growing cells in persistent infections is well documented for bacteria and has gained attention in parasitic protozoa in the last 10-15 years. Here, we show that, relative to their blood counterparts, the slender forms residing in the adipose tissue (ATFs) divide more slowly and synthetize less proteins, two hallmarks of persistence. This existence of persister-like cells in $T$. brucei infections may be important for disease chronicity and could contribute to treatment failure.

\section{Dynamic infection modeling predicts slow growing parasites}

Our model analysis adds an extra layer of complexity to the published models, typically focused on antigenic variation or differentiation mechanisms ${ }^{10,13,16,30}$. Here we addressed the existence of differences between parasite dynamics in different in-host compartments, namely the blood and adipose tissue, anticipating an adipose tissue population that replicates 2 -fold more slowly than the blood population, which is consistent with the temporal and magnitude trends present in the data.

Besides testing for inter-compartmental variability, this modeling approach also provides estimates for other relevant parameters involved in infection, such as the migration rate between 
compartments and contribution to clearance by the immune response. Model 2, for instance, predicts a migration rate of about $0.11 \mathrm{cell} / \mathrm{smg} /$ day suggesting that, per milligram of organ, each day about $11 \%$ of blood parasites leave to the adipose tissue and vice-versa ( 0.005 per hour). This observation is in line with previously estimated migration rates between blood and organs of mice infected with Salmonella enterica $(0.005 \text { per hour })^{31}$. Similarly, the lower bound for the ratio between slender vs stumpy cells killing rates, estimated by the joint posterior of this model in the interval 6.5-24, also falls within the reported estimated range $5-7^{32}$, although we find more uncertainty with this dataset. The remaining parameter estimates are similar between models and approximate existing values in the literature (e.g. antigen switching rate) $)^{13,30}$.

Naturally, the space for modeling is infinite, but including additional complexity is a stepwise process, ideally backed by different and incremental sources of data. Having found support for one major difference between the two compartments, does not rule out the existence of other sources of variation that may, on their own, or synergistically, contribute to further individuation of infection dynamics between the blood and adipose tissue. Future models, based on more-detailed infection time-series data (e.g. explicit antigenic variants, more time points for parasite densities, tissue-specific immune responses), combined with proteomics and highresolution microscopy, should be developed. This would allow assess to other dimensions of blood and adipose tissue variations, and integrate them in frameworks for parasite fitness in natural transmission settings ${ }^{33}$. Expanding the description of variant-specific traits or time-dependent phenotypes, such as gradual immune suppression, could help capture more accurately the intricacies of peak-to-peak variability that the current modeling framework does not account for. It would also be interesting to characterize other extravascular populations, by modelling other relevant and inter-linked in-host compartments such as the brain and the skin, and quantify their thus far-unexplored role in chronic infection.

\section{Cell-cycle remodeling and metabolic switch}

Persisters are defined as slow-growing or growth-arrested cells, with a metabolism adapted to the available nutrients and their energetic demands ${ }^{2}$. This phenotype may arise stochastically or in response to environmental cues such as nutrient limitation. When nutrient resources are not optimal, some bacteria remodel their metabolism and reduce their growth and protein synthesis, to become persisters. Persisters have been described in bacterial populations including Mycobacterium, Streptococcus, Staphylococcus and Candida ${ }^{34}$. Reports on protozoan parasites are more recent and less detailed but vast enough to accommodate different species like $T$. cruzi, Leishmania spp., Plasmodium spp. and T. gondii ${ }^{2}$. Adapting to the mammalian host microenvironments by acquiring persistence is a common strategy between parasites.

Inside the mammalian host, $T$. brucei colonizes several organs, including the adipose tissue, pancreas, skin and brain ${ }^{5,9}$. The proteomic analysis showed metabolic changes in ATFs resembling the ones observed in persistent parasitic protozoa, suggesting that the adipose tissue population could harbor persistent parasites. First, and foremost, the protein synthesis machinery is downregulated, while the pathway that produces ATP and acetate from pyruvate is upregulated. The observed increase of the purine salvage pathway in ATFs may reflect a need to increase the ATP production capacity in the adipose tissue environment. Interestingly, ATFs appears to share some features with stumpy forms, while it also shows important differences. Like stumpy forms, ATFs appear to show an increased production of ATP, via the acetate pathway ${ }^{35}$ and an upregulation of the enzymes of the glutamine/proline degradation pathway ${ }^{36,37}$. Unlike stumpy forms, ATFs did not show evidence for upregulation of the Protein Associated with Differentiation 1 (PAD1), a major hallmark of stumpy forms. The characteristic upregulations of Expression Site Associated Gene 9 (ESAG9), Protein Phosphatase 1 (PP1) and RNA-binding Protein 7 (RBP7) in stumpy forms were also not observed in the ATFs proteome ${ }^{38}$.

Another evidence that supports that ATFs may be persister cells is that the division time of ATFs is twice longer than BSFs. Reduced cell growth is the first evidence for the presence of persister cells in a population, consistent among bacterial and protozoan persisters ${ }^{2}$. Recently, Ward et al. reported the existence of $T$. cruzi persistent slow grower cells in the colon of chronically infected mice ${ }^{39}$. Also, T. gondii, one of the most widespread protozoan parasites, owes its persistent infection nature to metabolically active, transient slow grower persistent bradyzoites inhabiting tissue cysts as a strategy to face nutrient limitations and host immune pressure ${ }^{3}$.

In T. brucei life cycle, two stages consist of parasites in which cell cycle has been arrested: the stumpy and metacyclic forms. Exit from cell cycle arrest only takes place when those parasites 
have been transmitted to the new hosts, the Tsetse and the mammal, respectively ${ }^{6,7}$. In ATFs, we did not find evidence for a complete cell cycle arrest but rather a reduced growth. Moreover, in contrast to stumpy and metacyclic forms, ATFs can recolonize a new microenvironment within the mammalian host $^{8}$, indicating that the persistent behavior of ATFs is reversible within the mammalian host.

The extended presence of $T$. brucei parasites in the adipose tissue suggests that this reservoir might play a role in infection. Parasite evasion by migration to extravascular spaces together with persister-like phenotype may be a strategic protection from the immune response ${ }^{2}$, allowing for periodical blood repopulation. Alternatively, the reduced growth might be a consequence of a metabolic adaptation to the tissue environment, which could ultimately be associated to the wasting syndrome characteristic of trypanosomiasis in humans and cattle ${ }^{40}$. In the future, it would be interesting to test the metabolic and replicative behavior of parasites that colonize other tissues and test whether the persistence-behavior is specific to the adipose tissue.

Similar to what has been proposed for dormant $T$. gondii and persistent $L$. donovani cells, among other parasitic protozoa ${ }^{2}$, the proteome of $T$. brucei adipose tissue parasites also showed upregulation of proteins involved in oxidative stress and downregulation of proteins involved in protein synthesis. Intriguingly, the latter has been pointed as one of the explanations for drug resistance in persistent cells $\mathrm{s}^{2,41}$. It is tempting to speculate that $T$. brucei adipose tissue forms may be more resistant to drugs and contribute to relapses. Drug resistance has been a major concern in African Trypanosomiasis treatment given the observed relapses in treated patients ${ }^{42,43}$. The reasons for these observations are still a matter of debate in the literature. Our observation of persistent-like cells among the adipose tissue population of parasites (and possibly in other colonized tissues as well) could contribute to explain treatment failure, just as it does so in other persistent bacteria and parasitic infections. Novel efficient strategies for drug therapies must be based on, and incorporate improved knowledge of these persistent cell populations, especially their mechanisms, as a way to overcome their refractoriness and promote sterile cure.

\section{Methods}

\section{Parasite Cell Lines}

To characterize the infection dynamics, a $T$. brucei pleomorphic stumpy reporter cell line was used (AnTat1.1 GFP.:PAD1 utr) while for the remaining experiments, a monomorphic strain was selected (Lister 427), to avoid the mixture of the two life cycle stages (slender and stumpy forms). Procyclic forms (PCFs) were obtained from Lister 427 cultured parasites, by placing 2 million parasites in $1 \mathrm{~mL}$ of DTM medium with $6 \mathrm{mM}$ of cis-aconitic acid at $27^{\circ} \mathrm{C}$ with $5 \% \mathrm{CO}_{2}$ and allowing them to grow for 3 days.

\section{Animal Infections and Parasites Isolation}

Animal experiments were performed according to EU regulations and approved by the Órgão Responsável pelo Bem-estar Animal (ORBEA) of Instituto de Medicina Molecular and the competent authority Direcção Geral de Alimentação e Veterinária (license number: 018889 12016). Mice were group-housed in filter-top cages in a Specific-Pathogen-Free barrier facility under standard laboratory conditions: 21 to $22^{\circ} \mathrm{C}$ and a $12 \mathrm{~h}$ light/12 h dark cycle. Chow and water were available ad libitum. All infections were performed in 8-11 week old wild-type male C57BL/6J mice with origin in Charles River, France, by intraperitoneal (i.p.) injection of 2000 parasites, unless otherwise stated. Parasite viability was evaluated prior to infection under an optical microscope.

Parasites were collected from mice sacrificed by $\mathrm{CO}_{2}$ narcosis. Blood parasites were collected first by heart puncture, while adipose tissue parasites collection was performed on perfused gonadal depots incubated in HMI11 (or Creek's Minimal Medium, for the proteomics assay) at $37^{\circ} \mathrm{C}$ for up to 70 minutes. Mice were manually transcardially perfused with pre-warmed heparinized saline buffer (50mL 1X phosphate buffered saline (1X PBS) with $250 \mu \mathrm{L}$ of $5000 \mathrm{I} . \mathrm{U} . / \mathrm{mL}$ heparine per animal). Subsequently, parasites were isolated using a DEAE sepharose TM Fast Flow column whenever necessary (GE Healthcare).

\section{Infection Dynamics}


Parasitemia was assessed daily by taking blood from the mouse-tail vein and counting the number of parasites using a Neubauer chamber. The total number of parasites present in the blood and the gonadal depot was determined by qPCR. Briefly, animals were sacrificed by $\mathrm{CO}_{2}$ narcosis and blood collected by heart puncture. Blood sample was split into two. On one sample, red blood cells were lysed with Red Blood Cell Lysis Buffer ( $0.15 \mathrm{M}$ ammonium chloride; $0.01 \mathrm{M}$ potassium bicarbonate; $0.001 \mathrm{M}$ EDTA disodium salt) and the remaining pellet snap frozen in liquid nitrogen. The other sample was used for parasites isolation. After perfusion, gonadal depots were collected and one was snap frozen immediately while the other was used to isolate fat parasites. Genomic DNA (gDNA) was extracted from tissues using NZY tissue gDNA isolation kit (NZYTech, Portugal) and the amount of $18 \mathrm{~S}$ rDNA gene of $T$. brucei present in the blood and the gonadal depots was measured by qPCR and converted into number of parasites as described in ${ }^{8}$. Isolated parasites from blood and gonadal adipose tissue were then fixed and their GFP expression was analyzed on a BD LSRFortessa ${ }^{\mathrm{TM}}$ cell analyzer and data treated using FlowJo.

\section{Mathematical Modeling}

Average parasite densities and percentage of stumpy forms obtained from both blood and adipose tissue gonadal depot during the infection dynamics characterization were used to fit the mathematical model. The ordinary differential equations for population dynamics were based on ${ }^{13}$, but in this study the number of compartments was doubled to represent parasites growing in the blood and adipose tissue, and the immune response dynamics was simplified. The model variables for each antigenic wave were the slender and stumpy cells in the two compartments, as well as the variant-specific immune response. Allowing for up to five consecutive antigenic waves during 28 days resulted in 25 inter-dependent equations. Thus, infection processes were captured with a parsimonious formulation (growth, density-dependent differentiation, antigenic variation and antigen-dependent host immune response). Further, the two in-host compartments, blood and adipose tissue, were connected through migration, assumed to occur at the same rate in either direction and for all cells. All variants were assumed symmetric, in their parameters, including the switch rate to the next wave. The majority of parameters were estimated by fitting the model to the dynamic data; with exception of a few such as, the stumpy cell lifespan which was fixed at about 2 days $^{13}$ (Supplementary Model Information for detailed description on model structure and biological assumptions).

Model fitting to data was performed under a Bayesian framework, using the adaptive Monte Carlo Markov chain mcmcstat package in Matlab ${ }^{44}$. To compare hypotheses, Deviance Information Criterion (DIC) $)^{14}$, the posterior error distribution and marginal likelihood ${ }^{15}$ were computed for each model, and the quality of fits to data and feasibility of parameters were inspected.

\section{Proteomics}

Mice infected for 5 days were sacrificed and parasite isolation from blood and adipose tissue was performed as described above. Parasites were washed in Creeks Minimal Medium depleted from Fetal Bovine Serum and lysed with 1X LDS Buffer supplemented with $100 \mathrm{mM}$ Dithiothreitol (DTT) by boiling at $80^{\circ} \mathrm{C}$ for 15 minutes. Parasites isolated from up to 6 mice were pooled to obtain a minimum of 0.32 million parasites. Protein samples were then separated, prepared and measured as $\mathrm{in}^{45}$ except for the peptide elution. This step was performed with a 200 minutes optimized gradient from 2 to $40 \%$ acetonitrile with $0.1 \%$ formic acid at a flow rate of $225 \mathrm{~nL} / \mathrm{min}$. Protein quantification was processed in MaxQuant version 1.6.7.046 using standard settings. The raw proteomics files were searched against the protein databases of $T$. brucei TREU927 (TriTrypDB version 33), Mus musculus strain C57BL/6J (UniProt), the 14 VSGs of the Lister $427 \mathrm{~T}$. brucei strain (UniProt) and the contaminants database included in MaxQuant. Contaminants, reverse Protein Groups (PGs) and PGs only identified by a modification site or by less than 2 peptides (of which 1 needed to be unique) were removed as well as $M$. musculus identified proteins. To assign a quantification to missing values, these were imputed 1000 times using a $\beta$-distribution with equal shape parameters $(\alpha=\beta=2)$ and a PG was only considered differentially regulated if it was found up or downregulated in at least $99 \%$ of times. For each individual replicate, the obtained distribution was scaled between 0.1 and 1.5 percentile of the log2 transformed measured label-free quantitation (LFQ) intensity values. Finally, only the PGs that were quantified by LFQ intensity in at least 2 replicates of one condition (ATFs or BSFs) were considered for further analysis. Regulated PGs were determined if the relationship between the 
significance of the Welch's t-test and their fold-change was above a threshold defined by a reciprocal function (with limits $p$-value $=0.05$ and fold change $=1.5$ ).

The GO term enrichment analysis was performed in R with GO.db annotation package ${ }^{47}$ from Bioconductor and Fisher's Exact Test ( $p$-value $\leq 0.05$ ) from stats package. T. brucei TREU927 GO term annotations were obtained from TriTrypDB and GO term enrichment was assessed. PGs were associated with the GO terms of the individual genes in the group and a GO term enrichment test was performed separately on upregulated and downregulated gene. Only the GO terms with at least 5 annotated PGs were considered.

The mass spectrometry proteomics data have been deposited to the ProteomeXchange Consortium via the PRIDE partner repository with the dataset identifier PXD014958.

\section{Protein Synthesis}

Differences in protein synthesis were determined through Click-i ${ }^{\circledR}$ Homopropargylglycine (HPG) incorporation into nascent proteins. To establish the ideal concentration of HPG, cultured Lister 427 parasites were washed in methionine free Minimal Essential Medium and incubated in the same medium with $25 \mu \mathrm{M}, 50 \mu \mathrm{M}$ or $100 \mu \mathrm{M}$ of $\mathrm{HPG}$ for 30 minutes at $37^{\circ} \mathrm{C}$. Cultured parasites were also incubated in the same medium with $100 \mu \mathrm{g} / \mathrm{mL}$ of cycloheximide for 20 minutes at $37^{\circ} \mathrm{C}$ before adding 50 $\mathrm{MM}$ HPG. Isolated BSFs and ATFs as well as PCFs were also incubated with $50 \mu \mathrm{M}$ HPG under the same conditions. After incubation, all tested parasites were fixed with $1 \%$ paraformaldehyde and permeabilized with $0.5 \%$ Triton $\mathrm{X}-100.100 \mu \mathrm{L}$ of the reaction cocktail were then added for a 30-minute period at room temperature (light protected) and samples washed after with $100 \mu \mathrm{L}$ of Reaction Rinse Buffer (Click-iT ${ }^{\circledR}$ HPG Alexa Fluor ${ }^{\circledR} 488$ Protein Synthesis Assay Kit). All samples were stained with $1 \mu \mathrm{g} / \mathrm{mL}$ of DAPI and both DAPI and HPG intensities measured with BD LSRFortessa X-20. Data analyses were performed in FlowJo. Statistical significances were determined by Wilcoxon signed-rank test in the free software R: http://www.r-project.org.

\section{Cell-cycle Profile}

The number of kinetoplasts and nuclei were assessed for both blood and adipose tissue populations by microscopy imaging in vivo and ex vivo. To compare the two profiles, parasites from a pool of 2 mice infected for 5 days were stained either by intravenous injection of $10 \mathrm{mg} / \mathrm{kg} \mathrm{of}$ Hoechst $\mathrm{H} 33342$ (in vivo experiments) or by adding $1 \mu \mathrm{g} / \mathrm{mL}$ of Hoechst $\mathrm{H} 33342$ (ex vivo experiments) and observed by intravital microscopy and ex vivo microscopy, respectively. More than 600 cells were analyzed per condition.

\section{DNA synthesis}

Measurement of DNA synthesis was based on the incorporation of 5-ethynyl-2'deoxyuridine (EdU) and its subsequent detection by a fluorescent azide through "click" chemistry. EdU was either administered intravenously $(200 \mathrm{mg} / \mathrm{kg}$ ) to mice infected for 5 days (in vivo protocol), or added $(100 \mu \mathrm{M})$ to parasites isolated from mice infected for the same period and cultured in $\mathrm{HMI} 11$ at $37^{\circ} \mathrm{C}$ (ex vivo protocol). In both conditions, parasites were incubated with EdU for 30 minutes. The remaining protocol was conducted mostly according to manufacturer's instructions with some minor changes (ThermoFisher Scientific). Briefly, parasites were washed with cold 1xTDB, fixed with $1 \%$ paraformaldehyde for 10 minutes followed by quenching with $0.125 \mathrm{M}$ glycine for 5 minutes. Parasites were adhered to silanized coverslips for 30 minutes, permeabilized with $0.5 \%$ Triton X-100 for 20 minutes, washed twice with 3\%BSA in 1xPBS and fluorescently labeled with Alexa Fluor ${ }^{\circledR} 488$ fluorescent azyde by exposure to $300 \mu \mathrm{L}$ of Click-iT ${ }^{\circledR}$ reaction cocktail for an extra 30 minutes, protected from light. Finally, cells were washed twice with $3 \%$ BSA in 1xPBS, stained with $1 \mu \mathrm{g} / \mathrm{mL}$ of Hoechst $\mathrm{H} 33342$ for 30 minutes and the percentage of EdU positive cells was assessed by fluorescence microscopy. All the reported steps were performed at room temperature. More than 300 cells were analyzed per condition.

\section{Cell Proliferation}

10 million cultured parasites were labelled with $2 \mu \mathrm{M}$ of CellTrace ${ }^{\mathrm{TM}}$ Violet in $10 \mathrm{~mL} 1 \mathrm{xPBS}$ for 20 minutes at $37^{\circ} \mathrm{C}$, protected from light. Remnants of free dye were removed by adding $5 \mathrm{~mL}$ of $\mathrm{HMI} 11$ to the cells for 5 minutes at $37^{\circ} \mathrm{C}$. Pelleted parasites were then resuspended in $\mathrm{HMI} 11$ and 1 million immediately intraperitoneally injected in a mouse. A fraction of these parasites was fixed with $1 \%$ paraformaldehyde for 10 minutes followed by quenching with $0.125 \mathrm{M}$ glycine for 5 minutes and pellet resuspended in 3\%BSA/0.05\% TX-100 in 1xPBS and observed by microscopy 
to determine basal die incorporation. Two days post-infection, the Mean Fluorescence Intensity (MFI) levels of CTV within blood and adipose tissue parasites were assessed either by intravital microscopy or by ex vivo microscopy analyses. More than 180 cells were analyzed per condition. The doubling time was estimated by obtaining the number of divisions of each population $(x)$ from the slope of the CTV intensity versus the number of divisions $(y=C 0 e-0.693 x$, where $y$ is the determined mean fluorescence intensity of the analyzed population and $\mathrm{CO}$ is initial mean fluorescence intensity of the population used to infect mice) and then dividing the infection time by the obtained number of divisions (x).

\section{Microscopy}

All imaging was performed on a 3i Marianas spinning disc confocal microscope (Intelligent Imaging Innovations) using a $63 \times$ objective lens (Plan-Apochromat, NA 1.40, oil immersion, Zeiss). Laser stacks $405(405 \mathrm{~nm})$ and $488(488 \mathrm{~nm})$ were used for visualizing kinetoplasts and nuclei (Hoechst) or CTV, and FITC-Dextran, respectively. To avoid photodamage, an average maximum laser power of $2 \mathrm{~mW}$, a gain value of 1 and an exposure of 100 milliseconds were selected. A time lapse of 5 seconds was acquired, with images obtained every 10 milliseconds. In addition, bright field imaging was performed for visualization of infected parasites in the blood monolayer and BSFs and ATFs ex vivo. Background correction was performed with internal controls (i.e. regions without parasites in CTV positive samples for CTV experiments and cytoplasmic regions of parasites for EdU experiments) and exogenous controls (i.e. parasites in CTV- or EdU-negative samples). Segmentation (CTV experiments) was based on the bright field (blood) or 488nm (adipose tissue and blood) detections, and mean fluorescence intensity (MFI) was calculated for each parasite based on the CTV signal $(405 \mathrm{~nm})$, Hoechst H33342 signal $(405 \mathrm{~nm})$, or Click-iT ${ }^{\mathrm{TM}}$-EdU signal $(488 \mathrm{~nm})$. Parasites were imaged across the full available sample using a "snake by rows" observation and acquisition approach covering the entire tissue/dish. For all tested conditions, a minimum of 50 parasites per sample was imaged in a minimum of 25 different fields of view. Acquired images were then analyzed in $\mathrm{Fiji}^{48}$ and llastik (https://www.ilastik.org).

\section{Sample Preparation for Microscopy}

Prior to intravital microscopy, mice were anaesthetized with $120 \mathrm{mg} / \mathrm{Kg}$ ketamine and $16 \mathrm{mg} / \mathrm{Kg}$ xylazine injected intravenously. FITC-Dextran 70kDa was also injected intravenously immediately preceding imaging to distinguish between blood vessels and tissue parenchyma. The gonadal adipose tissue was exposed through a small incision of $20-40 \mathrm{~mm}$ made on the lower abdominal region of the mouse and a temporal glass window (Merck rectangular coverglass, $100 \mathrm{~mm} \times 50 \mathrm{~mm}$ ) implanted for imaging. The mouse was then placed on the microscope stage, and the infected blood vessels from the adipose tissue and the adipose tissue per se, were imaged to assess a sample of the BSFs and ATFs population, respectively. This protocol was applied to both gonads. BSFs population was also assessed by imaging blood monolayers obtained from $2 \mu \mathrm{L}$ of collected blood, diluted in $200 \mu \mathrm{L}$ of 1 xPBS and placed on a petri dish. Non-motile parasites were excluded from the analysis.

For ex vivo microscopy, isolated ATFs and BSFs were concentrated into $200 \mu \mathrm{l}$ by centrifugation for 2 minutes at 10,000rcf. The $200 \mu \mathrm{l}$ were then transferred to a glass bottom dish (Matek Life Sciences, 60mm, No. 1.5 thickness) and imaged.

\section{Acknowledgments}

The authors thank Keith Matthews (University of Edinburgh) for providing AnTat1.1E clone, Christian Janzen (University of Wurzburg) for the GFP::PAD1 utr cell line, Ruy M. Ribeiro for useful discussions about the statistical analysis and Helena Pinheiro for drawing the diagram of the mathematical infection model. The authors would like to acknowledge the Rodents, Bioimaging and Flow Citometry Units of the Instituto de Medicina Molecular and all members of the L.M.F. lab. This work was supported by IC\&DT Programa de Actividades Conjuntas - ref. 016417 "Oneida", 771714 (ERC) and 029161 (FCT), SFRH/BPD/89833/2012 (FCT) to S.T, LT000047/2019-L (HFSP) and ALTF 1048-2016 (EMBO) to M.D.N, IMM/BI/83-2017 (FCT individual fellowship, project number PTDC/BIM-MET/4471/2014) to T.B.R, IMM/BI/54-2016 (FCT individual fellowship, project number PTDC/BIM-MET/4471/2014)to F.BE and CEECIND/03322/2108 (FCT) to L.M.F. 
661

662

663

664

665

666

667

668

669

670

671

672

673

674

675

676

677

678

679

680

681

682

683

684

685

686

687

688

689

690

691

\section{Author Contributions}

S.T, M.D.N, J.F, E.G, L.M.F designed research; S.T, M.D.N, M.C.Q, T.B.R, F.BE, F.BU, E.G performed experiments; S.T, M.D.N, M.C.S, M.D, F.BR, E.G, L.M.F analyzed data and S.T, F.BR, E.G, L.M.F wrote the paper.

\section{Competing Interests}

The authors declare no competing interests.

References

1. Fisher, R. A., Gollan, B. \& Helaine, S. Persistent bacterial infections and persister cells. Nat. Rev. Microbiol. 15, 453-464 (2017).

2. Barrett, M. P., Kyle, D. E., Sibley, L. D., Radke, J. B. \& Tarleton, R. L. Protozoan persister-like cells and drug treatment failure. Nat. Rev. Microbiol. 17, 607-620 (2019).

3. Watts, E. et al. Novel approaches reveal that toxoplasma gondii bradyzoites within tissue cysts are dynamic and replicating entities in vivo. mBio 6, 1-24 (2015).

4. Chen, N. et al. Fatty acid synthesis and pyruvate metabolism pathways remain active in dihydroartemisinin-induced dormant ring stages of plasmodium falciparum. Antimicrob. Agents Chemother. 58, 4773-4781 (2014).

5. Pereira, S. S., Trindade, S., De Niz, M. \& Figueiredo, L. M. Tissue tropism in parasitic diseases. Open Biol. 9, (2019).

6. Rico, E. et al. Bloodstream form pre-adaptation to the tsetse fly in Trypanosoma brucei. Front. Cell. Infect. Microbiol. 4, 1-15 (2013).

7. Christiano, R. et al. The proteome and transcriptome of the infectious metacyclic form of Trypanosoma brucei define quiescent cells primed for mammalian invasion. Mol. Microbiol. 106, 74-92 (2017).

8. Trindade, S. et al. Trypanosoma brucei Parasites Occupy and Functionally Adapt to the Adipose Tissue in Mice. Cell Host Microbe 19, 837-48 (2016). 
692 9. Niz, M. De, Bras, D., Pedro, M. \& Nascimento, A. M. Organotypic endothelial adhesion

693 molecules are key for Trypanosoma brucei tropism and virulence. (2021).

694 10. MacGregor, P., Savill, N. J., Hall, D. \& Matthews, K. R. Transmission stages dominate 695

696 trypanosome within-host dynamics during chronic infections. Cell Host Microbe 9, 310-8

11. Vanwalleghem, G., Morias, Y., Beschin, A., Szymkowski, D. E. \& Pays, E. Trypanosoma brucei growth control by TNF in mammalian host is independent of the soluble form of the

12. MacGregor, P., Szöőr, B., Savill, N. J. \& Matthews, K. R. Trypanosomal immune evasion, chronicity and transmission: an elegant balancing act. Nat. Rev. Microbiol. 10, 431 (2012).

702

13. Gjini, E., Haydon, D. T., Barry, J. D. \& Cobbold, C. A. Critical interplay between parasite differentiation, host immunity, and antigenic variation in trypanosome infections. Am. Nat.

705

706

707 708

709

$$
176,424-439 \text { (2010). }
$$

14. Spiegelhalter, D. J., Best, N. G., Carlin, B. P. \& Van Der Linde, A. Bayesian measures of model complexity and fit. J. R. Stat. Soc. Ser. B Stat. Methodol. 64, 583-616 (2002).

15. Wilks, S. S. The Large-Sample Distribution of the Likelihood Ratio for Testing Composite Hypotheses. Ann. Math. Stat. 9, 60-62 (1938).

16. Tyler, K. M., Higgs, P. G., Matthews, K. R. \& Gull, K. Limitation of Trypanosoma brucei parasitaemia results from density-dependent parasite differentiation and parasite killing by the host immune response. Proc. R. Soc. B Biol. Sci. 268, 2235-2243 (2001).

17. Dejung, M. et al. Quantitative Proteomics Uncovers Novel Factors Involved in Developmental Differentiation of Trypanosoma brucei. PLOS Pathog. 12, e1005439 (2016).

18. Van Hellemond, J. J., Opperdoes, F. R. \& Tielens, A. G. M. Trypanosomatidae produce acetate via a mitochondrial acetate:succinate CoA transferase. Proc. Natl. Acad. Sci. U. S. A. 95, 3036-3041 (1998). 
717 19. Mazet, M. et al. Revisiting the Central Metabolism of the Bloodstream Forms of

718 Trypanosoma brucei: Production of Acetate in the Mitochondrion Is Essential for Parasite

719 Viability. PLoS Negl. Trop. Dis. 7, 1-14 (2013).

720 20. Mochizuki, K. et al. The ASCT/SCS cycle fuels mitochondrial ATP and acetate production in

721 Trypanosoma brucei. Biochim. Biophys. Acta - Bioenerg. 1861, 148283 (2020).

722 21. Johnston, K. et al. Mapping the metabolism of five amino acids in bloodstream form

723 Trypanosoma brucei using U-13C-labelled substrates and LC-MS. Biosci. Rep. 39, 1-17

724 (2019).

725 22. Capewell, P. et al. Regulation of Trypanosoma brucei Total and Polysomal mRNA during

726 Development within Its Mammalian Host. PLOS ONE 8, (2013).

727 23. Zhang, M., Joyce, B. R., Sullivan, W. J. \& Nussenzweig, V. Translational control in

728 Plasmodium and Toxoplasma parasites. Eukaryot. Cell 12, 161-167 (2013).

729 24. Martin, J. L. et al. Metabolic Reprogramming during Purine Stress in the Protozoan Pathogen

730 Leishmania donovani. PLoS Pathog. 10, (2014).

731 25. T. Sherwin and K. Gull. The cell division cycle of Trypanosoma brucei brucei timing of event

732 markers and cytoskeletal modulations. Philos Trans R Soc Lond B Biol Sci 323, 573-88

733 (1989).

734 26. Simeonov, A. A chemical method for fast and sensitive detection of DNA synthesis in vivo:

735 Commentary. Assay Drug Dev. Technol. 6, 157-158 (2008).

736 27. Lyons, A. B. \& Parish, C. R. Determination of lymphocyte division by flow cytometry. J.

737 Immunol. Methods 171, 131-137 (1994).

738 28. Haanstra, J. R. et al. Proliferating bloodstream-form Trypanosoma brucei use a negligible

739 part of consumed glucose for anabolic processes. Int. J. Parasitol. 42, 667-673 (2012).

740 29. Peacock, L., Ferris, V., Bailey, M. \& Gibson, W. Fly transmission and mating of Trypanosoma

741 brucei brucei strain 427. Mol. Biochem. Parasitol. 160, 100-106 (2008). 
30. Lythgoe, K. A., Morrison, L. J., Read, A. F. \& Barry, J. D. Parasite-intrinsic factors can explain ordered progression of trypanosome antigenic variation. Proc. Natl. Acad. Sci. U. S. A. 104, 8095-100 (2007).

31. Grant, A. J. et al. Modelling within-host spatiotemporal dynamics of invasive bacterial disease. PLoS Biol. 6, 757-770 (2008).

32. McLintock, L. M. L., Turner, C. M. R. \& Vickerman, K. Comparison of the effects of immune killing mechanisms on Trypanosoma brucei parasites of slender and stumpy morphology. Parasite Immunol. 15, 475-480 (1993).

33. Gjini, E., Haydon, D. T., Barry, J. D. \& Cobbold, C. A. Linking the antigen archive structure to pathogen fitness in African trypanosomes. Proc. R. Soc. B Biol. Sci. 280, (2013).

34. van den Bergh, B., Fauvart, M. \& Michiels, J. Formation, physiology, ecology, evolution and clinical importance of bacterial persisters. FEMS Microbiol. Rev. 41, 219-251 (2017).

35. Van Grinsven, K. W. A., Van Den Abbeele, J., Van Den Bossche, P., Van Hellemond, J. J. \& Tielens, A. G. M. Adaptations in the glucose metabolism of procyclic Trypanosoma brucei isolates from tsetse flies and during differentiation of bloodstream forms. Eukaryot. Cell 8, 1307-1311 (2009).

36. Naguleswaran, A., Doiron, N. \& Roditi, I. RNA-Seq analysis validates the use of culturederived Trypanosoma brucei and provides new markers for mammalian and insect life-cycle stages. BMC Genomics 19, 1-11 (2018).

37. Queiroz, R., Benz, C., Fellenberg, K., Hoheisel, J. D. \& Clayton, C. Transcriptome analysis of differentiating trypanosomes reveals the existence of multiple post-transcriptional regulons. BMC Genomics 10, 495 (2009).

38. Silvester, E., McWilliam, K. R. \& Matthews, K. R. The cytological events and molecular control of life cycle development of Trypanosoma brucei in the mammalian bloodstream. Pathogens 6, 1-19 (2017). 
770

771

772

773

774

775

776

777

778

779

780

781

782

783

784

785

786

787

788

789

790

791

39. Ward, A. I., Olmo, F., Atherton, R., Taylor, M. C. \& Kelly, J. M. Trypanosoma cruzi amastigotes have a reduced replication rate during chronic stage infections. bioRxiv (2020).

40. WHO. WHO Report on Global Surveillance of Epidemic-prone Infectious Diseases World. (2000).

41. Pontes, M. H. \& Groisman, E. A. A physiological basis for nonheritable antibiotic resistance. mBio 11, 1-13 (2020).

42. Pépin, J. \& Khonde, N. Relapses following treatment of early-stage Trypanosoma brucei gambiense sleeping sickness with a combination of pentamidine and suramin. Trans. R. Soc. Trop. Med. Hyg. 90, 183-186 (1996).

43. Richardson, J. B. et al. Whole genome sequencing shows sleeping sickness relapse is due to parasite regrowth and not reinfection. Evol. Appl. 9, 381-393 (2016).

44. Haario, H., Laine, M., Mira, A. \& Saksman, E. DRAM: Efficient adaptive MCMC. Stat. Comput. 16, 339-354 (2006).

45. Bluhm, A., Casas-Vila, N., Scheibe, M. \& Butter, F. Reader interactome of epigenetic histone marks in birds. Proteomics 16, 427-436 (2016).

46. Cox, J. \& Mann, M. MaxQuant enables high peptide identification rates, individualized p.p.b.-range mass accuracies and proteome-wide protein quantification. Nat. Biotechnol. 26, 1367-1372 (2008).

47. A. Alexa and J. Rahnenfuhrer. Enrichment Analysis for Gene Ontology. topGO R package version 2.24.0. (2016).

48. Schindelin, J. et al. Fiji: An open-source platform for biological-image analysis. Nat. Methods 9, 676-682 (2012). 
A.

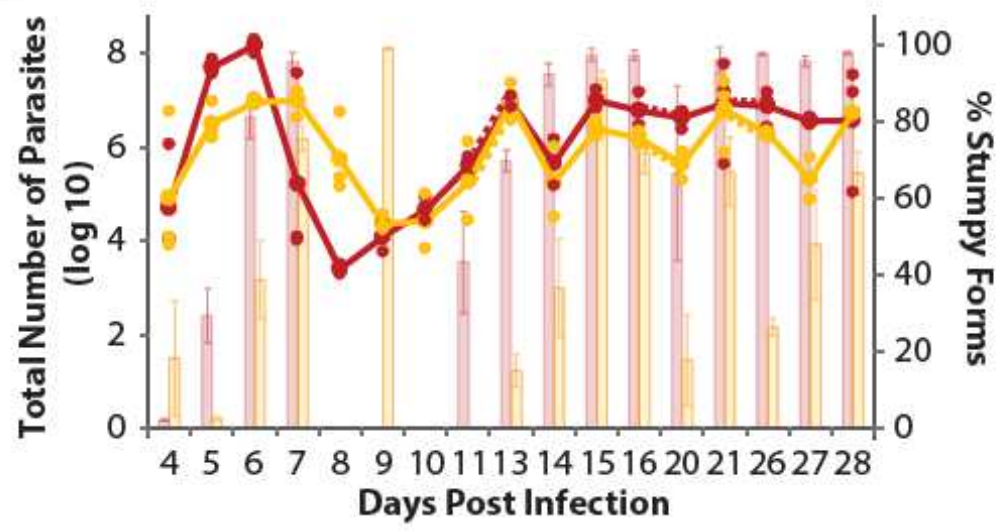

Blood: $\quad$ Adipose Tissue:

-Average \# Parasites Average \# Parasites

- \# Parasites per Mouse - \# Parasites per Mouse

$\square$ Stumpy Forms $\quad \%$ Stumpy Forms

B.

Figure 1. Infection dynamics in blood and gonadal adipose tissue.

A. C57BL/6J mice were infected with a pleomorphic T. brucei GFP.:PAD1 utr stumpy reporter cellline. The total number of parasites in each tissue was quantified by QPCR (red and yellow lines and dots) and the percentage of stumpy forms was estimated by FACS as the proportion of GFPpositive parasites (red and yellow bars). Data for each day of infection was obtained from a pool of 2-9 mice ( $n=2-4$ independent experiments per time point). The dashed lines correspond to noncontiguous time points and the full lines to contiguous time points. On days 8-11, with two exceptions, it was not possible to measure the proportion of stumpy forms due to a decrease in parasite load. B. Percentage of stumpy forms in blood (red) and gonadal adipose tissue (yellow) for the 17 studied time points. Each point represents one independent measurement (either from a single mouse or from a pool of up to 3 mice). Grey lines represent the average percentage of stumpy forms and error bars the standard deviation. C. Percentage of stumpy forms in blood and gonadal adipose tisue of five mice sacrificed at different days of infection $(5,7,14,21$ and 28). 
A.

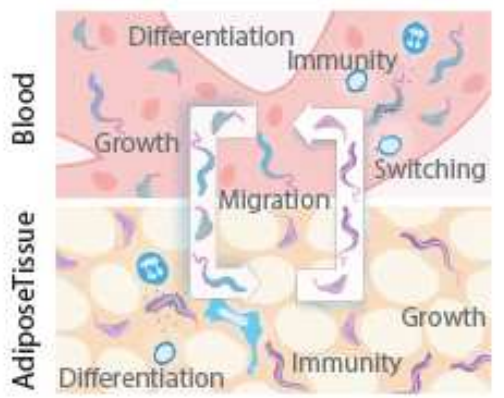

B.
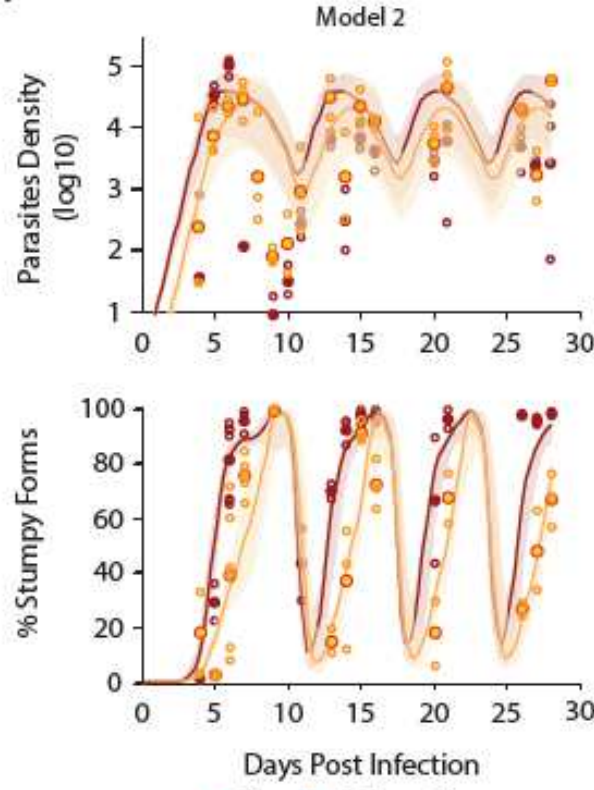

n Blood Adipose Tissue

Figure 2. Mathematical model for parasite dynamics in blood and gonadal adipose tissue. A. Diagram of the mathematical infection model. In this model, two compartments were considered: blood and adipose tissue. In each compartment, parasites may have intrinsic growth and differentiation rates. A constant migration rate was also considered between the two. The specific immune response was assumed to be triggered due to overall antigen stimulation from blood and adipose tissue parasites, after which it grows in response to increasing pathogen density and reaches each compartment (Materials and Methods and Supplementary Model Information). B. Model fit for predictions of parasite density and proportion of stumpy forms, in blood and adipose tissue, for the favored model (Model 2). Scattered empty circles indicate all observations from individual time-points and filled circles indicate the mean over replicates used to fit the model. The lines indicate the model prediction with mean parameters (as in Table 1). The shaded regions indicate the $95 \%$ credible envelopes from 50 simulations with random parameter combinations from the estimated posteriors. 
A.

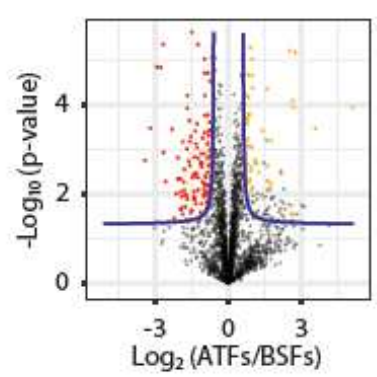

B.

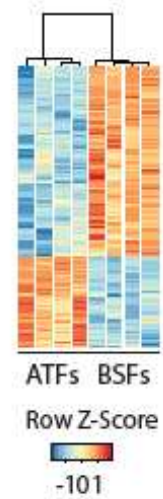

C.

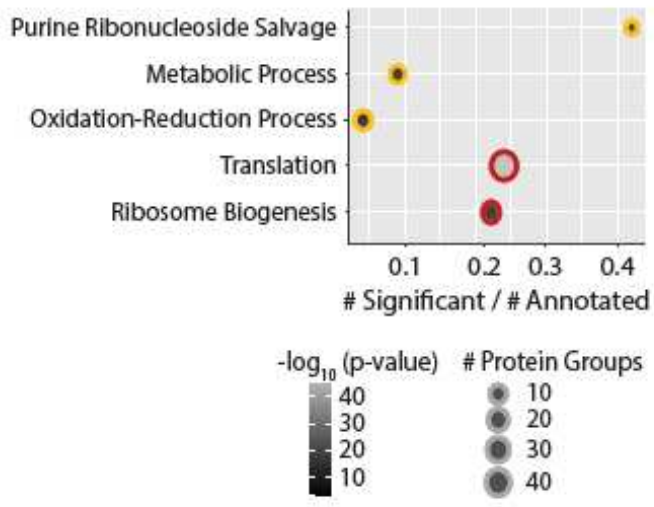

D.

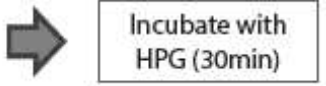

E.

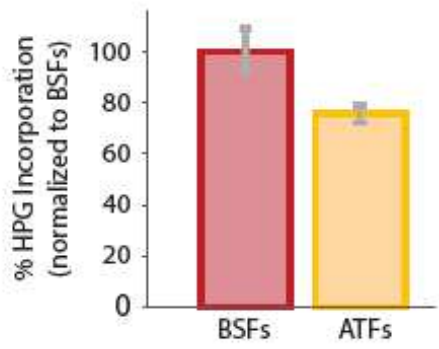

Figure 3. Proteome analysis and protein synthesis of parasites isolated from blood and gonadal adipose tissue.

A. Representative volcano plot of 1 out of the 1000 performed imputations with the 2693 protein groups detected by mass spectrometry in the sum of BSFs and ATFs 8 data sets. Red and yellow dots indicate protein groups significantly upregulated in BSFs and ATFs respectively. Proteins were obtained from Lister 427 parasites isolated from a pool of 5-6 animals infected for 5 days $(n=4$ independent experiments). B. Heat map representing the z-score abundances of the differentially expressed protein groups. Each column corresponds to one independent pool of mice. C. Enriched GO terms (Biological Process) of the differentially upregulated (yellow circles) and downregulated (red circles) proteins according to gene ratio, using a False Discovery Rate corrected p-value cutoff of 0.05 (Fisher's exact test). Each dot corresponds to a given GO term: the size represents the number of differentially expressed protein groups and the color the respective p-value. $\mathbf{D}$. Fluxogram of the experimental procedure used to quantify protein synthesis in Lister 427 parasites isolated from blood and gonadal adipose tissue 5 days post-infection. E. Percentage of HPG internalized by ATFs (yellow bar) normalized to the percentage of internalization of the BSFs (red bar) ( $n=6$ independent experiments). Error bars represent the Standard Error of the Mean. F. Mean Fluorescence Intensity of the HPG incorporated for 30 minutes by the isolated BSFs and ATFs for experiments A, B, C, D; E and $F$ and for the average of the six experiments $(n=6$ independent experiments). 
A.
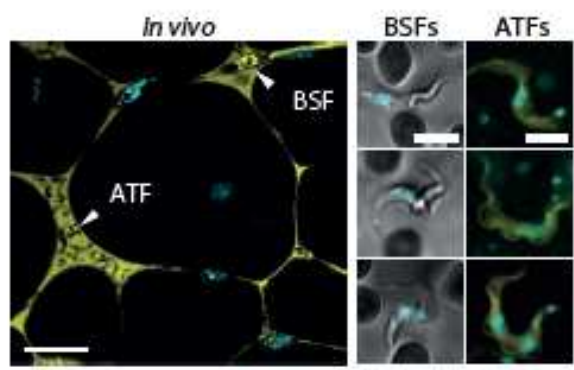

B.

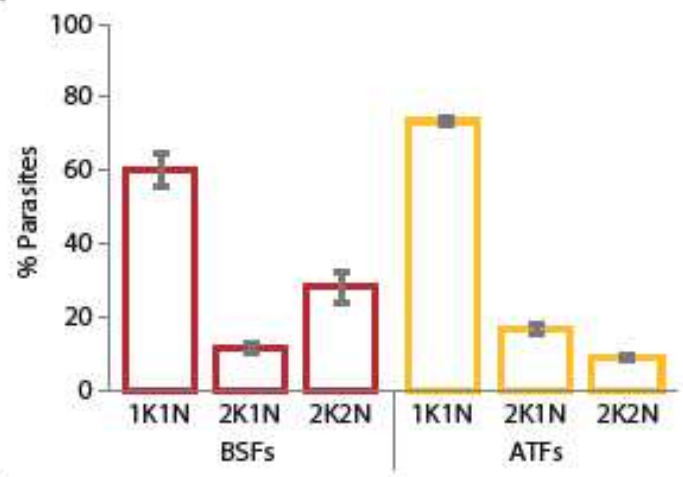

C.

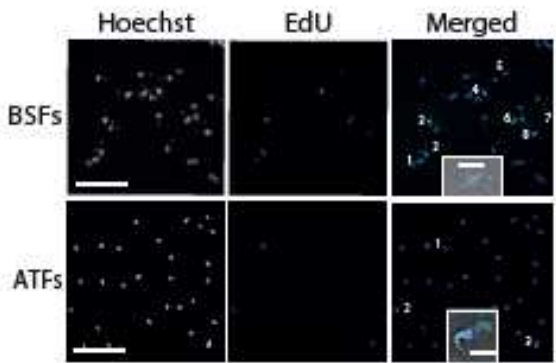

D.

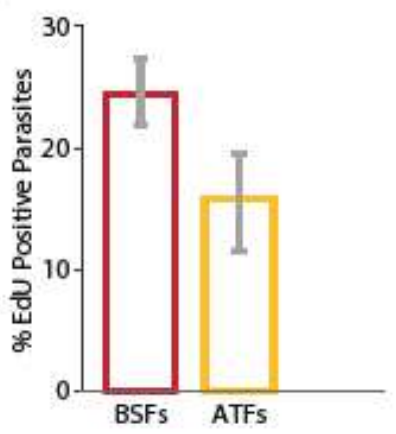

E.
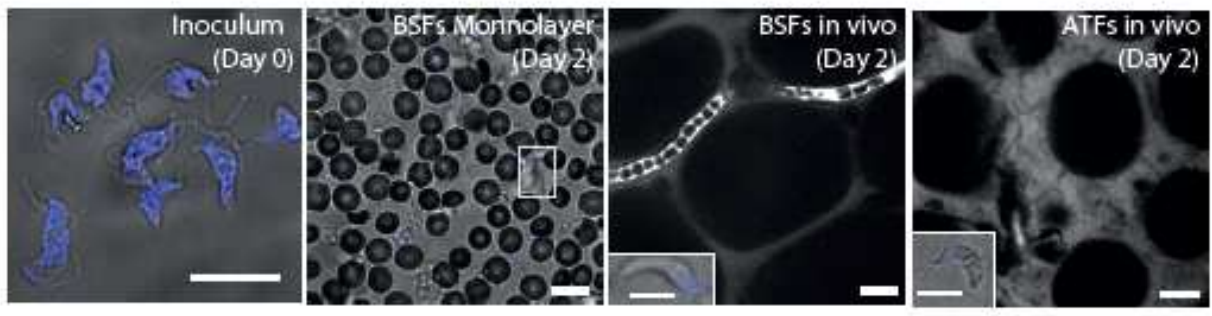

F.

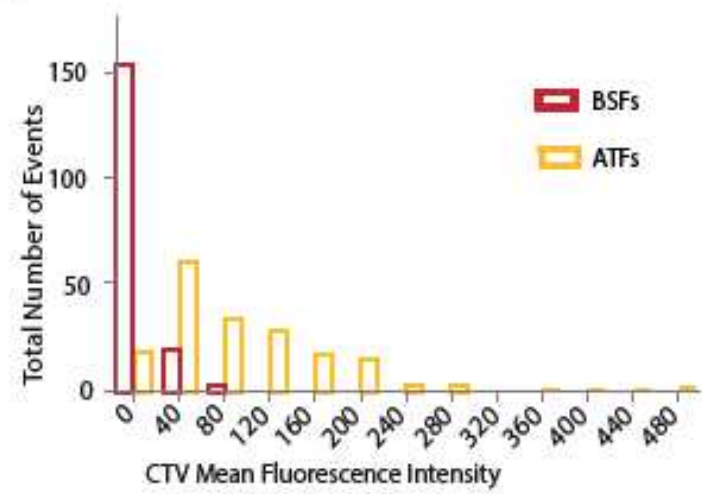

G.

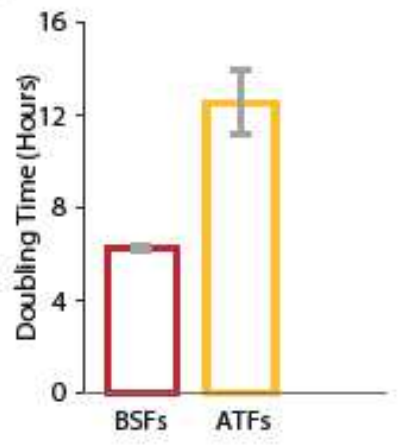

Figure 4. Parasite proliferation in blood and gonadal adipose tissue.

A. Cell-cycle analysis assayed by the number of Kinetoplasts $(K)$ and Nuclei $(N)$ in BSFs and ATFs Lister 427 parasites from mice infected for 5 days $(n=4$ independent experiments). Left side: Representative image of in vivo microscopy of the adipose tissue and a vessel (scale bar, $20 \mu \mathrm{m}$ ); Right side: representative images of the three parasites analyzed on a monolayer (BSFs) and in vivo (ATFs) (Hoechst staining, blue; scale bar, $5 \mu \mathrm{m}$ ). B. Quantification of cell cycle analysis. Percentage of parasites containing $1 \mathrm{~K} 1 \mathrm{~N}$ (cells in $\mathrm{G} 1$, early $\mathrm{S}$ phase or $\mathrm{G} 0$-arrested cells), $2 \mathrm{~K} 1 \mathrm{~N}$ (G2 and mitotic cells) and 2K2N (post-mitotic). More than 800 cells per condition. C. DNA synthesis assayed by the incorporation in vivo of EdU. Ex vivo microscopy images of Lister 427 BSFs and ATFs carrying recently synthesized DNA labelled with EdU ( $n=5$ independent experiments). 5 days 
861 post-infection, $200 \mathrm{mg} / \mathrm{kg}$ of EdU were administered intravenously and, 30 minutes later, mice were 862 sacrificed and the parasites isolated, labeled with Alexa Fluor ${ }^{\circledR} 488$ in a Click-it reaction, and 863 analyzed by microscopy. In the merged images, the EdU staining is shown by the Alexa Fluor 488 864 (green), which colocalizes with the nucleus and the kinetoplast, both stained with Hoechst (blue)

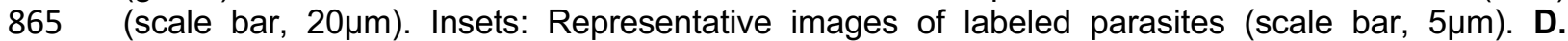
Quantification of DNA synthesis assay. Percentage of BSFs and ATFs that incorporated the thymidine analogue $(\mathrm{EdU})$, representing the proportion of parasites that were in $S$ phase or entered $\mathrm{S}$ phase during the 30 minutes pulsing period. More than 300 cells per condition. E. Doubling-time assayed by CellTrace ${ }^{\mathrm{TM}}$ Violet (CTV) staining dynamics. Representative images of ex vivo microscopy showing the inoculum of CTV labelled parasites (blue), a monolayer of BSFs stained

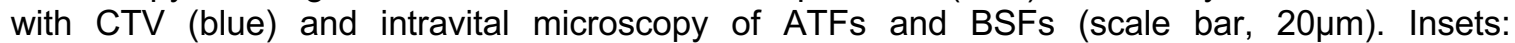
Representative images of labeled parasites (scale bar, $5 \mu \mathrm{m}$ ). F. Distribution of the CTV Mean Fluorescence Intensity of blood and adipose tissue parasites assessed by intravital microscopy ( $n=183$ BSFs and $n=199$ ATFs pooled from the 4 independent experiments). G. Quantification of CTV assay. Doubling time of BSFs and ATFs from mice infected for 2 days $(n=4$ independent experiments). Doubling time estimated for BSFs and ATFs considering the decrease in CTV signal intensity during 2 days. Doubling time was assessed by intravital microscopy of BSFs and ATFs from mice infected with cultured Lister 427 parasites pre-labeled with $2 \mu \mathrm{M}$ of CTV for 20 minutes immediately prior to infection. More than 180 cells per condition. 
Table 1. Mathematical Models Parameters and Estimates

883

\begin{tabular}{|c|c|c|c|c|c|}
\hline \multirow[t]{2}{*}{ Parameter } & \multirow[t]{2}{*}{ Interpretation } & \multicolumn{3}{|c|}{ Estimates $^{1}$} & \multirow[t]{2}{*}{ Units } \\
\hline & & Model 1 & Model 2 & Model 3 & \\
\hline$r$ & Growth Rate & $\begin{array}{l}2.12\left(7.8^{*}\right) \\
(1.85 ; 2.40)\end{array}$ & - & & Divisions/Per Day \\
\hline$r_{b}$ & Growth Rate of Slender Forms in Blood & - & $\begin{array}{l}2.38\left(7.0^{*}\right) \\
(2.10 ; 2.56)\end{array}$ & $\begin{array}{l}2.33\left(7.1^{*}\right) \\
(2.14 ; 2.49)\end{array}$ & Divisions/Per Day \\
\hline$r_{f}$ & Growth Rate of Slender Forms in Adipose Tissue & - & $\begin{array}{c}1.21\left(13.7^{*}\right) \\
(1.01 ; 1.56)\end{array}$ & $\begin{array}{c}1.23\left(13.5^{*}\right) \\
(1.01 ; 1.69)\end{array}$ & Divisions/Per Day \\
\hline$d$ & Minimal Killing Rates for Slender Forms ${ }^{2}$ & $\begin{array}{c}0.0016 \\
(0.0001 ; 0.0063)\end{array}$ & $\begin{array}{c}0.0032 \\
(0.0005 ; 0.0066)\end{array}$ & $\begin{array}{c}0.0029 \\
(0.0006 ; 0.0065)\end{array}$ & Cells/mg/Day \\
\hline$d^{\prime}$ & Minimal Killing Rates for Stumpy Forms ${ }^{2}$ & $\begin{array}{c}0.0005 \\
(0.0001 ; 0.0028)\end{array}$ & $\begin{array}{c}0.0001 \\
(0.00005 ; 0.0008)\end{array}$ & $\begin{array}{c}0.0002 \\
(0.00005 ; 0.0009)\end{array}$ & Cells/mg/Day \\
\hline$K$ & Density for Maximal Differentiation & $\begin{array}{c}12600 \\
(3400: 51000)\end{array}$ & $\begin{array}{c}31300 \\
(6700: 58700)\end{array}$ & - & Cells/mg \\
\hline$K_{b}$ & Density for Maximal Differentiation in Blood & $\begin{array}{c}(3400: 31000) \\
-\end{array}$ & $\begin{array}{c}16 / 00.38 / 001 \\
-\end{array}$ & $\begin{array}{c}26400 \\
(4000 ; 58100)\end{array}$ & Cells/mg \\
\hline$K_{f}$ & Density for Maximal Differentiation in Adipose Tissue & - & - & $\begin{array}{c}14200 \\
(3300 ; 54200)\end{array}$ & Cells/mg \\
\hline$k$ & Density for Half-Saturation Immune Stimulation & $\begin{array}{c}830 \\
(210 ; 6440)\end{array}$ & $\begin{array}{c}3600 \\
(990 ; 16600)\end{array}$ & $\begin{array}{c}3400 \\
(1200 ; 14000)\end{array}$ & Cells/mg \\
\hline$\sigma$ & Activation Rate of Anti VSG Immune Response & $\begin{array}{c}1.38 \\
(1.04 ; 1.83)\end{array}$ & $\begin{array}{c}1.68 \\
(1.27 ; 2.21)\end{array}$ & $\begin{array}{c}1.67 \\
(1.28 ; 2.21)\end{array}$ & Per Day \\
\hline$\mu$ & Migration Rate Across Compartments & $\begin{array}{c}0.0117 \\
(0.0069 ; 0.0311)\end{array}$ & $\begin{array}{c}0.1075 \\
(0.0523 ; 0.1901)\end{array}$ & $\begin{array}{c}0.0633 \\
(0.0101 ; 0.8353)\end{array}$ & Cells/mg/Day \\
\hline$s$ & Switch Probability to Next Antigenic Wave in Blood & $\begin{array}{c}0.0007 \\
(0.0001 ; 0.0051)\end{array}$ & $\begin{array}{c}0.0004 \\
(0.0001 ; 0.0014)\end{array}$ & $\begin{array}{c}0.0006 \\
(0.0002 ; 0.0019)\end{array}$ & Per Division \\
\hline
\end{tabular}

${ }^{1}$ Mean $(95 \% \mathrm{Cl})$

${ }^{2}$ Number of killed cells by 1 unit of immune response due to the feedback from VSG-specific immunity

* Doubling time (hours) 


\section{Supplementary Files}

This is a list of supplementary files associated with this preprint. Click to download.

- TrindadeSupplementarylnformation.pdf

- TrindadeSupplementaryInformationMovieS1.avi

- TrindadeSupplementaryInformationDataset.xlsx 